\title{
Clinical Outcomes of Single-Step Transepithelial Photorefractive Keratectomy and Off-Flap Epipolis- Laser in Situ Keratomileusis in Moderate to High Myopia: 12-Month Follow-Up
}

\author{
Yunjie Zhang \\ Wenzhou Medical University \\ Tiankun Li \\ Wenzhou Medical University \\ Zhangliang Li \\ Wenzhou Medical University \\ Mali Dai \\ Wenzhou Medical University \\ Qinmei Wang \\ Wenzhou Medical University \\ Chenchen Xu ( $\nabla$ xcc@mail.eye.ac.cn ) \\ Wenzhou Medical University
}

\section{Research Article}

Keywords: transepithelial photorefractive keratectomy, epipolis-laser in situ keratomileusis, higher-order aberrations

Posted Date: December 6th, 2021

DOI: https://doi.org/10.21203/rs.3.rs-1130441/v1

License: (c) (i) This work is licensed under a Creative Commons Attribution 4.0 International License. Read Full License 


\section{Abstract \\ Background}

To compare the quantitative and qualitative optical outcomes of single-step transepithelial photorefractive keratectomy (TPRK) and off-flap epipolis-laser in situ keratomileusis (Epi-LASIK) in moderate to high myopia.

\section{Methods}

In this prospective self-control study, we included patients with moderate to high myopia who were randomized to undergo TPRK in one eye and Epi-LASIK in the other eye. Twelve-month follow-up results for visual acuity, refraction, ocular high-order aberrations, contrast sensitivity, postoperative pain, epithelial healing, and haze grade were assessed.

\section{Results}

A total of 64 eyes (32 patients) were enrolled in the study. More eyes completed re-epithelialization in the TPRK group than in the Off-flap Epi-LASIK group 3-4 days postoperatively, while all eyes completed reepithelialization by seven days. More eyes achieved a visual acuity (both UDVA and CDVA) of better than $20 / 20$ in the TPRK group than in the Off-flap Epi-LASIK group. The $\pm 0.50 \mathrm{D}$ predictability for correction of the spherical equivalent (SE) was higher in the eyes of the TPRK group (91\%) than in those of the off-flap Epi-LASIK group (80\%) 12 months after surgery. No significant differences in ocular aberrations, including coma, spherical, and trefoil, were found between the two groups at 12 months. There were also no significant differences in visual acuity, contrast sensitivity, pain, and haze grading between the two groups.

\section{Conclusions}

Both TPRK and off-flap Epi-LASIK are safe, effective, and predictable treatments for moderate to high myopia with comparable surgical outcomes.

\section{Trial registration:}

This study was retrospectively registered on ClinicalTrial.gov (NCT05060094, 17/09/2021).

\section{Background}


Photorefractive keratectomy (PRK) and laser in situ keratomileusis (LASIK) were introduced decades ago $[1,2]$, and with recent advances in surgical techniques and adjunctive mitomycin-C medication, the outcome of modern surface ablation is excellent and comparable to that of LASIK $[3,4]$.

A key aspect of modern surface ablation is epithelial removal. Laser-assisted sub-epithelial keratectomy (LASEK), a technique which allows for the separation of the epithelium using $20 \%$ alcohol, was introduced in 1996 [5]. Then, in 2001, epipolis-laser in situ keratomileusis (Epi-LASIK) was developed as an alternative to epithelial separation using a modified microkeratome [6]. Recently, Feng et al. [7] demonstrated that complete removal of the epithelial flap in Epi-LASIK (off-flap) resulted in less postoperative pain, more rapid re-epithelialization, and faster visual recovery compared to flap-reserved Epi-LASIK (on-flap).

Another alternative technique for epithelial removal is transepithelial PRK (TPRK), in which epithelial removal is performed with laser phototherapeutic (PTK) ablation, followed by laser refractive ablation of the stroma. However, several studies have reported that two-step TPRK using different laser platforms produces variable results [8-11]. Excitingly, a single-step TPRK was recently introduced which allows for simultaneous ablation of both the epithelium and stroma which shortens treatment time, minimizes the risk of corneal dehydration, and prevents any instrument to eye contact. This new technique uses a population-based epithelial thickness ablation profile of $55 \mu \mathrm{m}$ centrally, and $65 \mu \mathrm{m}$ at $4 \mathrm{~mm}$ radially from the center to match the area of the total ablation zone. Considering that the water content of the epithelium is different from that of the stroma, the ablation rates of these two are also different. While previous studies have compared the outcomes between single-step TPRK and alcohol-assisted PRK, no study to date has assessed the outcomes between single-step TPRK and off-flap LASIK [12-14].

Therefore, in this study, we evaluated the long-term outcomes of single-step TPRK compared to off-flap LASIK in moderate to high myopia, including qualitative and quantitative parameters, and performed vector analysis of astigmatism.

\section{Methods}

\section{Patients and Methods}

Patients with myopia (up to $-9.75 \mathrm{D}$ ) with or without astigmatism (up to $3.5 \mathrm{D}$ ) were enrolled in a prospective interventional study. The study was approved by the Wenzhou Medical University Institutional Review Board and adhered to the tenets of the Helsinki Declaration. Patients received a full explanation of both procedures and provided written informed consent. Patients were randomized to have TPRK in one eye and Epi-LASIK in the other eye using a random number table generated by Microsoft Excel.

Inclusion criteria included: 18 years of age or older; a corrected distance visual acuity (CDVA) in logarithm of minimal angle of resolution (LogMAR) of 0.10 or better; refractive error stabilized for at least one year; and discontinuation of contact lens use for at least two weeks. Exclusion criteria included: the presence of corneal scars, keratoconus, glaucoma, retinal diseases, or a history of corneal or intraocular surgery. 


\section{Patient Assessment}

All patients underwent comprehensive ophthalmological examinations, including uncorrected (UDVA) and corrected distance visual acuities (CDVA), manifest refraction, intraocular pressure, an anterior and posterior segment examination, corneal topography measured by a Scheimpflug scanning-slit topographer (Pentacam, Oculus Optikgerate $\mathrm{GmbH}$ ), and contrast sensitivity under photopic and mesopic conditions (CSV-1000E, Vector Vision Inc., Greenville, OH, USA) with correction by spectacles and ocular wavefront aberrometry (OPD-Scan II, Nidek Co. Ltd). These examinations were repeated 1, 3, 6, and 12 months after surgery.

\section{Surgical Technique}

Both single-step TPRK and off-flap Epi-LASIK were performed with the Amaris $750 \mathrm{~S}$ (Schwind eye-techsolutions, Germany) using integrated ORK-CAM software, with the right eye operated on first in all cases. Local anesthetic was applied for the ablation procedure, using proparacaine hydrochloride $0.5 \%$ drops (Alcaine, Alcon, USA) three times every five min.

In the single-step TPRK procedure, transepithelial corneal epithelial removal was performed using the ORK-CAM software module mode followed by stromal excimer laser ablation in a single step based on an aberration-free and aspheric profile. The area of epithelial removal was defined as the area of the total ablation zone. The software compensated for the energy loss at the slope of the peripheral cornea. In the off-flap Epi-LASIK group, the epithelium was removed using an epikeratome (Moria, Antony, France) with a diameter of $9 \mathrm{~mm}$. After ablation, $0.02 \%$ mitomycin-C was applied to the stromal bed for $15-30 \mathrm{~s}$ and then rinsed with a balanced salt solution. A bandage contact lens (Acuvue Oasis, Johnson \& Johnson, USA) was placed on the cornea at the end of the surgery. All procedures were performed by the same surgeon.

\section{Postoperative Medication and Assessment}

Patients were prescribed levofloxacin $0.5 \%$ eyedrops (Cravit; Santen, Japan) and pranoprofen $0.1 \%$ eye drops (Senju Pharmaceutical Co., Ltd) four times a day for the first three days after surgery. After removing the contact lens, tobramycin-dexamethasone eye drops (Tobradex; Alcon, Ltd.) were administered four times a day for two weeks, followed by $0.1 \%$ fluorometholone eye drops (FML; Allergan, Irvine, CA) which were used four times a day for the next two weeks, after which they were tapered every week to once daily. Preservative-free artificial tears every two hours were prescribed until complete reepithelialization, and then reduced to four times a day for six months.

Epithelial healing was observed daily from the third postoperative day, and the contact lens was removed when corneal re-epithelialization was completed. Subjective pain scores were evaluated on the third day according to a predetermined scale ranging from $0-5$ as follows: 0 , no pain or discomfort; 1 , photophobia and tears; 2 , photophobia and tears with mild pain; 3 , photophobia and tears with moderate pain that does not require oral medication; 4 , photophobia and tears with severe pain that oral medication can 
relieve; 5 , photophobia and tears with severe pain that oral medication cannot relieve. The corneal haze grade was recorded according to the methods reported by Fantes et al. [15]: 0 , no haze; 0.5 , trace haze that could only be seen by oblique illumination; 1 , a more visible haze not interfering with the visibility of iris details; 2 , mild influence of iris details; 3 , moderate influence of iris details; 4 , marked haze obscuring the stroma of the ablation area.

\section{Statistical Analysis}

Statistical analysis was performed using SPSS software (version 22.0, SPSS, Chicago, Inc.). The Kolmogorov-Smirnov test was used to check the normal distribution of the variables. Student's t-test or Wilcoxon rank sum test was used based on the normality of the data. Statistical significance was set at $p$ $<0.05$.

\section{Results}

In total, 38 patients were initially enrolled in the study. Thirty-two patients finally completed the 12-month follow-up, and six patients were lost to follow-up. No significant difference was noted between the groups at baseline with respect to spherical equivalent (SE), CDVA, central corneal thickness (CCT), or epithelial thickness. The optical zones between the groups were not significantly different. However, the central ablation depth and total ablation zone were significantly higher in the TPRK group compared to the Offflap Epi-LASIK group (both $P<.001$, Table 1). 
Table 1. Baseline characteristics of eyes that had transepithelial PRK or off-flap Epi-LASIK.

\begin{tabular}{|c|c|c|c|c|c|}
\hline \multirow[b]{2}{*}{ Parameter } & \multicolumn{2}{|c|}{ Transepithelial PRK } & \multicolumn{2}{|c|}{ Off-flap Epi-LASIK } & \multirow{2}{*}{$\begin{array}{l}\mathrm{P} \\
\text { value }\end{array}$} \\
\hline & Mean \pm SD & Range & Mean \pm SD & Range & \\
\hline \multicolumn{6}{|l|}{ Refractive errors (D) } \\
\hline Sphere & $-6.03 \pm 1.97$ & $-9.50,-2.50$ & $-6.18 \pm 2.01$ & $-9.75,-2.50$ & .363 \\
\hline Cylindrical & $-1.09 \pm 0.72$ & $-3.00,0.00$ & $-1.03 \pm 0.73$ & $-3.50,0.00$ & .616 \\
\hline SE & $-6.57 \pm 2.01$ & $-10.00,-2.88$ & $-6.67 \pm 1.99$ & $\begin{array}{l}-10.25 \\
-2.88\end{array}$ & .519 \\
\hline UDVA (logMAR) & $1.07 \pm 0.22$ & $0.50,1.60$ & $1.08 \pm 0.23$ & $0.50,1.60$ & .730 \\
\hline CDVA (LogMAR) & $-0.003 \pm 0.05$ & $-0.10,0.10$ & $0.003 \pm 0.04$ & $-0.10,0.10$ & .423 \\
\hline CCT (um) & $498.78 \pm 23.81$ & $\begin{array}{l}465.00 \\
570.00\end{array}$ & $499.00 \pm 22.53$ & $\begin{array}{l}462.00 \\
556.00\end{array}$ & .789 \\
\hline Total ablation zone $(\mathrm{mm})$ & $7.95 \pm 0.32$ & $6.70,8.44$ & $7.47 \pm 0.26$ & $6.89,8.14$ & $<.001$ \\
\hline Optical zone (mm) & $6.30 \pm 0.32$ & $5.80,7.00$ & $6.30 \pm 0.35$ & $5.80,7.00$ & .872 \\
\hline $\begin{array}{l}\text { Stromal ablation depth } \\
\text { (um) }\end{array}$ & $154.56 \pm 24.09$ & $\begin{array}{l}99.00 \\
188.00\end{array}$ & $101.19 \pm 19.40$ & $\begin{array}{l}61.00 \\
129.00\end{array}$ & $<.001$ \\
\hline $\begin{array}{l}\text { SE= Spherical equivalent; } \\
\text { visual acuity; logMAR = los } \\
\text { thickness. }\end{array}$ & $\begin{array}{l}\mathrm{VA}=\text { uncorrecte } \\
\text { rithm of the min }\end{array}$ & $\begin{array}{l}\text { I distance visu } \\
\text { mum angle of }\end{array}$ & $\begin{array}{l}\text { acuity; } C D V A=c \\
\text { solution; } C C T=c\end{array}$ & $\begin{array}{l}\text { rrected dista } \\
\text { ntral corneal }\end{array}$ & \\
\hline
\end{tabular}

\section{Corneal Epithelial Healing and Pain Score}

More eyes completed re-epithelialization in the TPRK group three days (20 eyes, $63 \%$ vs. 16 eyes, 50\%) and four days ( 25 eyes, $81 \%$ vs. 24 eyes, $78 \%$ ) after surgery than in the off-flap Epi-LASIK group.

Nevertheless, all eyes underwent re-epithelialization by seven days. There was no significant difference in median pain score (range) between the two groups (TPRK: 2.0 [4] vs. Off-flap Epi-LASIK: 1.0 [5], $P=.372$ ).

\section{Visual Acuity, Efficacy, and Safety}

During the period of postoperative observation, more eyes achieved visual acuity (both UDVA and CDVA) better than 20/20 in the TPRK group than in the Off-flap Epi-LASIK group (shown in Fig. 1A). None of the eyes lost two lines or more of CDVA in either group at the 12-month follow-up, and $60.00 \%(n=18)$ of the eyes in the TPRK group and $63.33 \%(n=19)$ eyes in the off-flap Epi-LASIK group gained one or two lines of improved CDVA ( shown in Fig. 1B). There was no significant difference between the two groups in terms of line gain or loss $(P=.58)$. Furthermore, no significant differences in UDVA or CDVA were noted between the groups (shown in Fig. 1C). At 12 months postoperatively, there were no significant differences in the 
mean efficacy index (ratio of postoperative UDVA to preoperative CDVA), mean safety index (ratio of postoperative to preoperative CDVA), refractive error (sphere, cylinder, and SE), UDVA, and CDVA between the two groups (Table 2).

\begin{tabular}{|c|c|c|c|c|c|}
\hline \multirow[b]{2}{*}{ Parameter } & \multicolumn{2}{|c|}{ Transepithelial PRK } & \multicolumn{2}{|c|}{ Off-flap Epi-LASIK } & \multirow[t]{2}{*}{ P Value } \\
\hline & Mean \pm SD & Range & Mean \pm SD & Range & \\
\hline \multicolumn{6}{|c|}{ Refractive errors (D) } \\
\hline Sphere & $0.21 \pm 0.33$ & $-0.50,1.00$ & $0.33 \pm 0.46$ & $-0.50,1.25$ & .218 \\
\hline Cylindrical & $-0.43 \pm 0.30$ & $-1.00,0.00$ & $-0.48 \pm 0.39$ & $-1.50,0.00$ & .584 \\
\hline SE & $-0.01 \pm 0.35$ & $-0.88,0.88$ & $0.08 \pm 0.42$ & $-0.88,1.00$ & .311 \\
\hline UDVA (logMAR) & $-0.05 \pm 0.09$ & $-0.20,0.20$ & $-0.04 \pm 0.08$ & $-0.10,0.20$ & .326 \\
\hline CDVA (logMAR) & $-0.06 \pm 0.06$ & $-0.10,0.10$ & $-0.05 \pm 0.06$ & $-0.10,0.10$ & .522 \\
\hline Efficacy index & $1.13 \pm 0.16$ & $0.79,1.26$ & $1.11 \pm 0.19$ & $0.79,1.41$ & .733 \\
\hline Safety index & $1.15 \pm 0.12$ & $1.00,1.26$ & $1.14 \pm 0.13$ & $0.89,1.41$ & .631 \\
\hline
\end{tabular}

\section{Refraction}

The $\pm 0.50 \mathrm{D}$ predictability for correction of the spherical equivalent (SE) was higher in the eyes of the TPRK group (91\%) compared to those of the off-flap Epi-LASIK group (80\%) 12 months after surgery (shown in Fig. 1D). The linear regression model of the attempted vs. achieved SE refraction in the TPRK group had a slope and coefficient $\left(r^{2}\right)$ of 0.996 and 0.9717 , respectively, and 0.998 and 0.9594 , respectively, in the off-flap Epi-LASIK group (shown in Fig. 1E).

With respect to astigmatism correction, all treated eyes in the TPRK group and 31 treated eyes (97\%) in the off-flap Epi-LASIK group exhibited postoperative cylinders of $1.00 \mathrm{D}$ or less (shown in Fig. 1F). The linear regression analysis between the target-induced astigmatism (TIA) and surgically induced astigmatism (SIA) vectors revealed slopes and coefficients $\left(r^{2}\right)$ of, 0.87 , and 0.68 , respectively, in the TPRK group (shown in Fig. 2A) and 0.88, and 0.65, respectively, in the off-flap Epi-LASIK group (shown in Fig. 2B). Figure 3 shows the TIA and SIA vector polar diagrams of the TPRK group (shown in Fig. $3 A$ and $B$ ) and the off-flap Epi-LASIK group (shown in Fig. 3C and D), respectively. No significant differences were found between two groups, with respect to magnitude and axis of TIA, SIA, difference vector and the value of angle of error, correction index and index of success (Table 3 ). 


\begin{tabular}{|c|c|c|c|c|c|}
\hline \multirow[b]{2}{*}{ Parameter } & \multicolumn{2}{|c|}{ Transepithelial PRK } & \multicolumn{2}{|c|}{ Off-flap Epi-LASIK } & \multirow[t]{2}{*}{ P Value } \\
\hline & Mean \pm SD & Range & Mean \pm SD & Range & \\
\hline \multicolumn{6}{|l|}{ TIA } \\
\hline Magnitude (D) & $0.91 \pm 0.62$ & $0.00,2.67$ & $0.90 \pm 0.65$ & $0.00,3.06$ & .936 \\
\hline Axis (degrees) & $102.0 \pm 75.6$ & $1.0,180.0$ & $109.2 \pm 72.8$ & $1.0,180.0$ & .698 \\
\hline \multicolumn{6}{|l|}{ SIA } \\
\hline Magnitude (D) & $1.12 \pm 0.65$ & $0.25,2.75$ & $1.21 \pm 0.71$ & $0.21,3.06$ & .464 \\
\hline Axis (degrees) & $92.7 \pm 75.5$ & $1.1,180.0$ & $97.8 \pm 74.7$ & $1.0,179.2$ & .796 \\
\hline \multicolumn{6}{|l|}{ Difference vector } \\
\hline Magnitude (D) & $0.43 \pm 0.30$ & $0.00,1.00$ & $0.48 \pm 0.39$ & $0.00,1.49$ & .473 \\
\hline Axis (degrees) & $81.4 \pm 52.2$ & $0.00,170.0$ & $82.2 \pm 55.6$ & $0.00,175.0$ & .953 \\
\hline Angle of Error (degrees) & $-1.5 \pm 13.9$ & $-38.9,35.9$ & $2.2 \pm 13.2$ & $-49.0,72.1$ & .394 \\
\hline Correction index & $1.32 \pm 0.61$ & $0.44,3.19$ & $1.45 \pm 0.76$ & $0.50,3.90$ & .419 \\
\hline Index of success & $0.53 \pm 0.54$ & $0.00,1.99$ & $0.65 \pm 0.72$ & $0.00,3.05$ & .410 \\
\hline
\end{tabular}

\section{Ocular Wavefront Aberrations}

In the eyes of the TPRK group, there was a significant decrease in ocular spherical, coma, and trefoil aberrations (all $P$.027) 12 months after surgery. In the eyes of the off-flap Epi-LASIK group, there was a significant decrease in ocular spherical and trefoil aberrations (both $P$.012) 12 months postoperatively. The difference in ocular higher-order aberrations (HOAs) between the two groups was not significant at 12 months after surgery. By 12 months, the photopic and mesopic contrast sensitivity was not significantly different between the groups (Table 4). 
Table 4. Comparison of ocular higher-order aberrations and contrast sensitivity in eyes that had transepithelial PRK or off-flap Epi-LASIK.

\begin{tabular}{|c|c|c|c|c|c|}
\hline & \multicolumn{2}{|c|}{ Transepithelial PRK } & \multicolumn{2}{|c|}{ Off-flap Epi-LASIK } & \multirow[t]{2}{*}{ P Value } \\
\hline & Mean \pm SD & Range & Mean \pm SD & Range & \\
\hline \multicolumn{6}{|c|}{ HOAs (um) } \\
\hline Spherical & $0.053 \pm 0.120$ & $0.001,0.581$ & $0.077 \pm 0.206$ & $0.001,1.084$ & $.348^{*}$ \\
\hline Coma & $0.118 \pm 0.200$ & $0.007,0.963$ & $0.156 \pm 0.245$ & $0.007,1.069$ & $.209^{*}$ \\
\hline Trefoil & $0.148 \pm 0.205$ & $0.019,0.796$ & $0.142 \pm 0.150$ & $0.021,1.160$ & $.802^{*}$ \\
\hline \multicolumn{6}{|c|}{ CS photopic } \\
\hline $3 \mathrm{cpd}$ & $1.76 \pm 0.13$ & $1.49,1.93$ & $1.74 \pm 0.12$ & $1.49,2.08$ & .326 \\
\hline $6 \mathrm{cpd}$ & $2.03 \pm 0.16$ & $1.70,2.29$ & $2.02 \pm 0.17$ & $1.70,2.29$ & .670 \\
\hline $12 \mathrm{cpd}$ & $1.73 \pm 0.18$ & $1.40,1.99$ & $1.72 \pm 0.18$ & $1.40,1.99$ & .744 \\
\hline $18 \mathrm{cpd}$ & $1.31 \pm 0.18$ & $0.96,1.55$ & $1.30 \pm 0.18$ & $0.81,1.55$ & .689 \\
\hline \multicolumn{6}{|c|}{ CS mesopic } \\
\hline $3 \mathrm{cpd}$ & $1.73 \pm 0.13$ & $1.49,1.93$ & $1.70 \pm 0.11$ & $1.49,1.93$ & .155 \\
\hline $6 \mathrm{cpd}$ & $2.01 \pm 0.14$ & $1.70,2.29$ & $2.01 \pm 0.17$ & $1.70,2.29$ & 1.000 \\
\hline $12 \mathrm{cpd}$ & $1.69 \pm 0.19$ & $1.40,1.99$ & $1.68 \pm 0.20$ & $1.40,1.99$ & .714 \\
\hline $18 \mathrm{cpd}$ & $1.25 \pm 0.21$ & $0.81,1.55$ & $1.26 \pm 0.22$ & $0.96,1.55$ & .821 \\
\hline
\end{tabular}

\section{Haze}

We discovered that one eye had $0.5+$ haze after off-flap Epi-LASIK and two eyes had $0.5+$ haze after TPRK. In the Off-flap Epi-LASIK group, 0.5 + or 1.0 + haze was noted in 19 eyes (59.4\%), nine eyes (28.1\%), and four eyes (12.5\%) 1, 3, and 6 months postoperatively, respectively. In the TPRK group, $0.5+$ or 1.0 + haze was found in 17 eyes (53.1\%), 10 eyes (31.3\%), and six eyes (18.8\%) 1, 3, and 6 months postoperatively, respectively. There were no significant differences between the groups.

\section{Discussion}

In this self-controlled study, we investigated the surgical outcomes of TPRK and off-flap Epi-LASIK in patients with moderate to high myopia. The visual outcomes indicated that both procedures were safe 
and effective for the correction of moderate to high myopia. There were no significant differences in postoperative visual acuity, efficacy, and safety indices as well as photopic and mesopic contrast sensitivity between the two groups. The $\pm 0.50 \mathrm{D}$ predictability for correction of the SE refraction was higher in the TPRK group than in the Off-flap Epi-LASIK group 12 months after surgery. Additionally, no significant differences in ocular HOAs (including spherical, coma, and trefoil aberrations) were noted between the groups.

As for refractive and visual outcomes, both TPRK and off-flap Epi-LASIK had comparable efficacy and predictability in the correction of moderate to high myopia. This is in contrast to a study by Fadlallah et al. [13], which found that TPRK was safe to treat only mild to moderate myopia and easier to perform compared to alcohol-assisted PRK. This is probably because TPRK has been modified several times since its introduction in 2009. Another study by Arba et al. [21] reported that if the actual corneal epithelial thickness is thinner or thicker than the applied profile and the actual corneal center-to-periphery progression deviates from the ablated one, refractive complications may occur in TPRK. In our study, TPRK demonstrated refractive results comparable to those of off-flap Epi-LASIK. While the reason for this is unclear, it may be that the eyes involved in our study were normal, non-pathologic, and not previously operated on; thus, these corneas highly matched the population-based epithelial ablation profile.

Regarding corneal haze, no significant difference was noted at any postoperative interval. By six months postoperatively, no eyes in either group had a haze over the score of 0.5. Haze can be seen after surface ablation in most cases as a mild anterior stromal opacity. It is considered to be the product of the normal healing process, which peaks at three months and fades at six months [16]. Haze formation is mainly associated with deep ablation, discontinuation of topical steroids [17], and irregularity of the anterior stroma [18]. The adjunction of $0.02 \%$ mitomycin C (MMC) over the ablated stroma led to lower levels of haze compared to conventional PRK [5][19]. The application of $0.02 \% \mathrm{MMC}$ may explain why the eyes in these two groups did not experience severe haze.

In our study, TPRK eyes showed faster re-epithelialization compared to Epi-LASIK eyes, but no significant difference was noted which is similar to findings from previous studies. For example, Aslanides et al. [14] found that by day three, significantly more eyes that underwent TPRK $(97 \%)$ had epithelial recovery compared to the alcohol-assisted PRK eyes (57\%). Another study by Kaluzny et al. [20] used spectral OCT to evaluate the epithelium healing process in patients who underwent different procedures in their two eyes. They reported that the mean healing time was $2.4 \pm 0.8$ days in the TPRK group and $3.6 \pm 0.9$ days in the alcohol-assisted group. For the TPRK procedure, the epithelial ablation profile was based on the study by Reinstein et al. [21], which characterized the epithelial thickness of normal eyes. They found that this makes the ablation more precise, provides a smoother and more regular stromal surface as well as speeds up epithelium healing. Meanwhile, the diameter of the epithelial removal in TPRK matched the total ablation zone, which was significantly smaller than that in the off-flap Epi-LASIK $(9 \mathrm{~mm})$. This may explain why off-flap Epi-LASIK eyes needed a longer time to heal in our study. 
In reflecting the quality of vision in daily life situations, more attention should be paid to contrast sensitivity and wavefront aberrations rather than visual acuity alone. Ocular high-order aberrations significantly decreased 12 months after surgery in both groups, except for ocular coma in the eyes of the off-flap Epi-LASIK group. Interestingly, Aslanides et al.[14] reported a significant increase in spherical aberration postoperatively, but it was equivalent in both the TPRK and off-flap Epi-LASIK groups. Previous studies have demonstrated that surface ablation provides better visual quality than LASIK because it induces fewer HOAs than LASIK $[22,23]$. In fact, higher-order aberrations are usually related to glare, halo, and vision disturbances, especially in patients with a large pupil or those under scotopic conditions. However, ocular aberrations as well as the photopic and mesopic contrast sensitivities at 12 months postoperatively were not significantly different between the groups. Thus, we conclude that the final visual quality 12 months after surgery was comparable between the TPRK and off-flap Epi-LASIK.

The current study had some limitations. First, the sample size was relatively small. A large population size could increase the power to detect significant differences. Second, the follow-up duration was relatively short. Therefore, future studies incorporating a longer follow-up of visual performance between TPRK and off-flap Epi-LASIK are recommended.

\section{Conclusions}

We conclude that both TPRK and off-flap Epi-LASIK are safe, effective, and predictable for treating moderate to high myopia. These two types of surgical techniques have comparable epithelium healing time, pain scores, corneal haze grade, and visual outcomes.

\section{Abbreviations}

TransPRK= single-step transepithelial photorefractive keratectomy;

Epi-LASIK= epipolis laser in situ keratomileusis;

PRK= photorefractive keratectomy;

LASIK= Laser in situ keratomileusis;

LASEK= Laser-assisted sub-epithelial keratectomy;

CDVA = corrected distance visual acuity;

$\log M A R=$ logarithm of minimal angle of resolution;

UDVA= uncorrected distance visual acuities;

$\mathrm{CS}=$ contrast sensitivity;

CCT = central corneal thickness; 


\section{Declarations}

Ethics approval and consent to participate $\ T$ The study was approved by the review board of the Eye Hospital of Wenzhou Medical University and adhered to the tenets of the Declaration of Helsinki. Written informed consents to participate in the study were obtained from participants or participants' parent or legal guardian.

Consent for publication: Not applicable.

Availability of data and materials: The datasets used and/or analyzed during the current study are available from the corresponding author on reasonable request.

Competing interests $₫$ The authors have no proprietary or commercial interest in any materials discussed in this article.

Funding $\varangle$ This study was supported by the Natural Science Foundation of Zhejiang Province (LQ20H120002), Medical and Health Science and Technology Project of Zhejiang Province (2019RC221), XinMiao Project of Science and Technology Department of Zhejiang Province (2021R413013).

Authors' Contributions: $Y Z$ designed the study and was a major contributor in writing the manuscript; TL was a major contributor in writing the manuscript; ZL analyzed and interpreted the data; MD collected the data; QW made critical revision of manuscript; $C X$ made substantial contributions to the design of the work.

Acknowledgements: We would like to acknowledge Ruiwen Zhang and Shuangshuang Zhang's assistance in collecting patient information.

\section{References}

1. Trokel SL, Srinivasan R, Braren B. Excimer laser surgery of the cornea. Am J Ophthalmol. 1983.

2. McAlinden C, Skiadaresi E, Moore JE. Visual and refractive outcomes following myopic laser-assisted subepithelial keratectomy with a flying-spot excimer laser. J Cataract Refract Surg. 2011.

3. Wallau AD, Campos M. Photorefractive keratectomy with mitomycin C versus LASIK in custom surgeries for myopia: A bilateral prospective randomized clinical trial. J Refract Surg. 2008.

4. Shalaby A, Kaye GB, Gimbel H V. Mitomycin C in photorefractive keratectomy. J Refract Surg. 2009.

5. Scerrati E. Laser in situ keratomileusis vs. laser epithelial keratomileusis (LASIK vs. LASEK). In: Journal of Refractive Surgery. 2001.

6. Pallikaris IG, Katsanevaki VJ, Kalyvianaki MI, Naoumidi II. Advances in subepithelial excimer refractive surgery techniques: Epi-LASIK. Current Opinion in Ophthalmology. 2003. 
7. Feng YF, Chen SH, Stojanovic A, Wang QM. Comparison of clinical outcomes between "On-Flap" and "Off-Flap" Epi-LASIK for myopia: A meta-analysis. Ophthalmologica. 2011.

8. Buzzonetti L, Petrocelli G, Laborante A, Mazzilli E, Gaspari M, Valente P, et al. A new transepithelial phototherapeutic keratectomy mode using the NIDEK CXIII excimer laser. J Refract Surg. 2009.

9. Muller LT, Candal EM, Epstein RJ, Dennis RF, Majmudar PA. Transepithelial phototherapeutic keratectomy/photorefractive keratectomy with adjunctive mitomycin-C for complicated LASIK flaps. J Cataract Refract Surg. 2005.

10. Ghadhfan F, Al-Rajhi A, Wagoner MD. Laser in situ keratomileusis versus surface ablation: Visual outcomes and complications. J Cataract Refract Surg. 2007.

11. Lee HK, Lee KS, Kim JK, Kim HC, Seo KR, Kim EK. Epithelial healing and clinical outcomes in excimer laser photorefractive surgery following three epithelial removal techniques: Mechanical, alcohol, and excimer laser. Am J Ophthalmol. 2005.

12. Fadlallah A, Fahed D, Khalil K, Dunia I, Menassa J, El Rami H, et al. Transepithelial photorefractive keratectomy: Clinical results. J Cataract Refract Surg. 2011.

13. Luger MHA, Ewering T, Arba-Mosquera S. Consecutive myopia correction with transepithelial versus alcohol-assisted photorefractive keratectomy in contralateral eyes: One-year results. J Cataract Refract Surg. 2012.

14. Aslanides IM, Padroni S, Mosquera SA, loannides A, Mukherjee A. Comparison of single-step reverse transepithelial all-surface laser ablation (ASLA) to alcohol-assisted photorefractive keratectomy. Clin Ophthalmol. 2012.

15. Fantes FE, Hanna KD, Waring GO, Pouliquen Y, Thompson KP, Savoldelli M. Wound Healing After Excimer Laser Keratomileusis (Photorefractive Keratectomy) in Monkeys. Arch Ophthalmol. 1990.

16. Lee YG, Chen WYW, Petroll WM, Cavanagh HD, Jester J V. Corneal haze after photorefractive keratectomy using different epithelial removal techniques: Mechanical debridemen versus laser scrape. Ophthalmology. 2001.

17. Caubet E. Cause of subepithelial corneal haze over 18 months after photorefractive keratectomy for myopia. Refract Corneal Surg. 1993.

18. Netto M V., Mohan RR, Sinha S, Sharma A, Dupps W, Wilson SE. Stromal haze, myofibroblasts, and surface irregularity after PRK. Exp Eye Res. 2006.

19. Bedei A, Marabotti A, Giannecchini I, Ferretti C, Montagnani M, Martinucci C, et al. Photorefractive keratectomy in high myopic defects with or without intraoperative mitomycin C: 1-Year results. Eur J Ophthalmol. 2006.

20. Kaluzny BJ, Szkulmowski M, Bukowska DM, Wojtkowski M. Spectral OCT with speckle contrast reduction for evaluation of the healing process after PRK and transepithelial PRK. Biomed Opt Express. 2014.

21. Reinstein DZ, Archer TJ, Gobbe M, Silverman RH, Coleman DJ. Epithelial thickness in the normal cornea: Three-dimensional display with artemis very high-frequency digital ultrasound. J Refract Surg. 2008. 
22. Yang $X$, Wang Y, Zhao K, Fang L. Comparison of higher-order aberration and optical quality after EpiLASIK and LASIK for myopia. Graefe's Arch Clin Exp Ophthalmol. 2011.

23. Moshirfar M, Schliesser JA, Chang JC, Oberg TJ, Mifflin MD, Townley R, et al. Visual outcomes after wavefront-guided photorefractive keratectomy and wavefront-guided laser in situ keratomileusis: Prospective comparison. J Cataract Refract Surg. 2010;36:1336-43. doi:10.1016/j.jcrs.2010.02.012.

\section{Figures}

\section{Figure 1}

Visual outcomes after single-step transepithelial photorefractive keratectomy (TPRK) and off-flap epipolis-laser in situ keratomileusis (Epi-LASIK). A: cumulative 12-month postoperative uncorrected distance visual acuity (UDVA) and corrected distance visual acuity (CDVA); Changes in the Snellen lines of postoperative UDVA (B) and CDVA (C), compared with preoperative CDVA; $D$ : accuracy of spherical equivalent refraction; $\mathrm{E}$ : attempted versus achieved changes in spherical equivalent refraction; $\mathrm{F}$ : distribution of preoperative and 12-month postoperative cylinder.
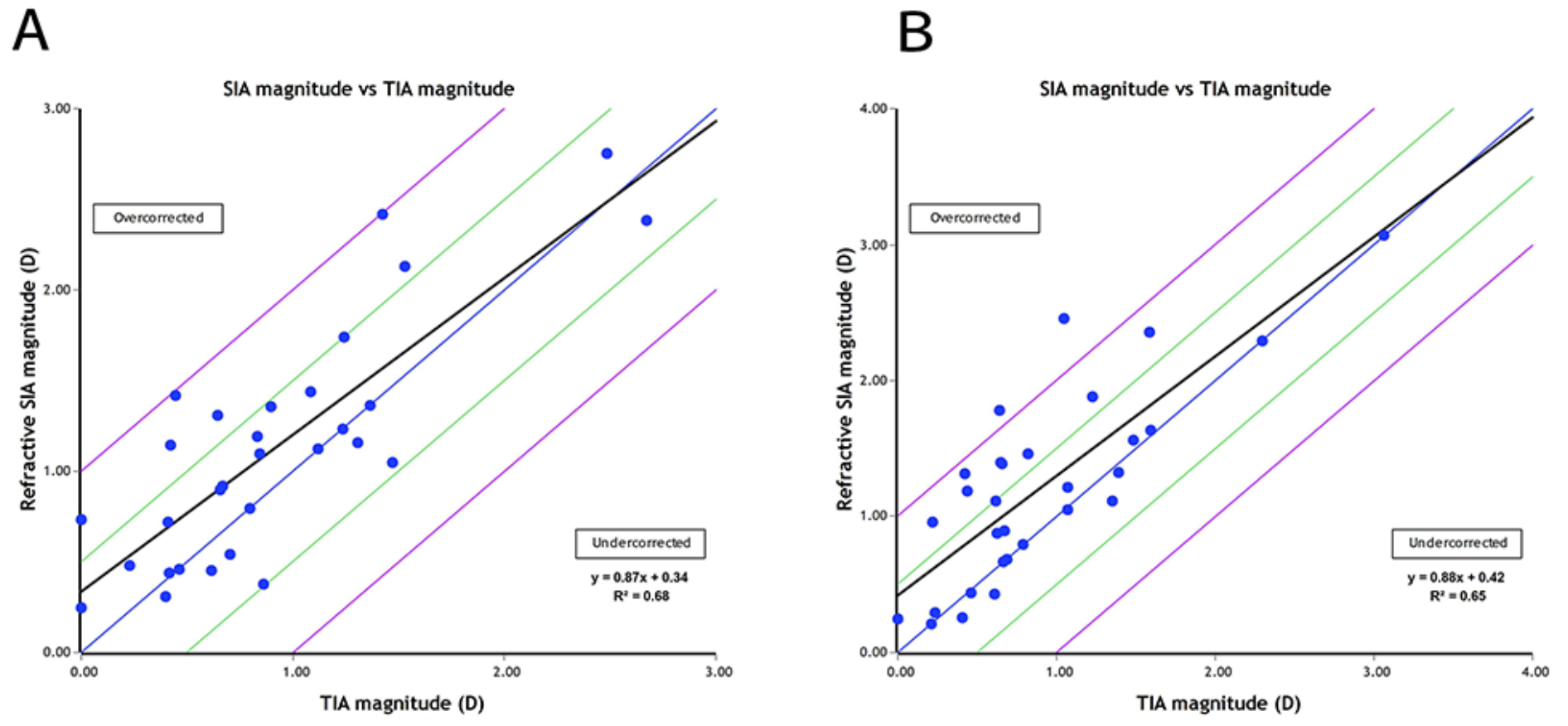

Figure 2

Target-induced versus surgically-induced astigmatism vectors at 12 months postoperatively.

Figure 3 
Single-angle polar plots of the target-induced astigmatism vector and surgically induced astigmatism at 12 months postoperatively in the single-step transepithelial photorefractive keratectomy (TPRK) group $(A, B)$ and off-flap epipolis-laser in situ keratomileusis (Epi-LASIK) group (C,D), respectively. 